Int. J. Dev. Biol. 50: 113-122 (2006)

doi: $10.1387 / \mathrm{ijdb} .052057 \mathrm{lb}$

\title{
Gastrulation in amphibian embryos, regarded as a succession of biomechanical feedback events
}

\author{
LEV V. BELOUSSOV*, NATALIA N. LUCHINSKAYA, ALEXANDER S. ERMAKOV \\ and NADEZHDA S. GLAGOLEVA \\ Faculty of Biology, Moscow State University, Moscow, Russia
}

\begin{abstract}
Gastrulation in amphibian embryos is a composition of several differently located morphogenetic movements which are perfectly coordinated with each other both in space and time. We hypothesize that this coordination is mediated by biomechanical interactions between different parts of a gastrulating embryo based upon the tendency of each part to hyper-restore the value of its mechanical stress (see Beloussov and Grabovsky, 2006). The entire process of gastrulation in amphibian embryos is considered as a chain of these mutually coupled reactions, which are largely dependent upon the geometry of a given embryo part. We divide gastrulation into several partly overlapped steps, give a theoretical interpretation for each of them, formulate the experiments for testing our interpretation and describe the experimental results which confirm our point of view. Among the predicted experimental results are: inhibition of radial cell intercalation by relaxation of tensile stresses at the blastula stage; inversion of convergent intercalation movements by relaxation of circumferential stresses at the early gastrula stage; stress-promoted reorientation of axial rudiments, and others. We also show that gastrulation is going on under a more or less constant average value of tensile stresses which may play a role as rate-limiting factors. A macromorphological biomechanical approach developed in this paper is regarded as complementary to exploring the molecular machinery of gastrulation.
\end{abstract}

KEY WORDS: gastrulation, morphogenesis, mechanical stresses, Xenopus laevis

It is not birth, marriage or death, but gastrulation
which is truly the most important time in your life.

L. Wolpert (1986)

\section{Introduction}

Gastrulation in general and, particularly in amphibian embryos (taking the Anurans and, in particular, Xenopus laevis as examples) is a space/temporal composition of several morphogenetic events. The main ones are: (1) radial cell intercalation $(\mathrm{RCl})$ in the blastocoel roof; (2) formation of bottle-shaped cells in the marginal zone, starting from the dorsal side of an embryo; (3) convergent cell intercalation $(\mathrm{CCl})$ in the suprablastoporal zone (SBZ) directed towards dorso-medial embryo midline (Keller, 1987; Keller and Danilchik, 1988; Wilson et al., 1989); (4) involution around the blastoporal lip (Holtfreter, 1944; Keller, 1981, 1984; Keller and Hardin, 1987). Although it has long been known (Spemann, 1936; Holtfreter, 1944; Keller and Jansa, 1992) that all of these processes can proceed, in experimental conditions, independently from each other, their rates, locations and direc- tionality are, after such an isolation, largely distorted. Meanwhile, in an entire embryo they are perfectly coordinated and enhance each other. Moreover, gastrulation-like movements can be easily reproduced in small pieces of embryonic tissues (Holtfreter, 1944) which shows that they are not unambiguously related to any definite regional properties of embryo material. That makes it possible that the gastrulation movements are the components of a structurally stable chain of self-organizing events linked with each other by some kinds of feedback. In this study we use the theoretical constructions described in the accompanying paper (Beloussov and Grabovsky, this volume) and suggest that the feedback may be based upon the tendency of any part of an embryo which is mechanically affected (stretched, relaxed or compressed) by other part(s) to hyper-restore its initial stress value, reciprocally affecting other part(s). We divide the entire

Abbreviations used in this paper: CCI, convergent cell intercalation; CE, contraction-extension (feedback); EE, extension-extension (feedback); HR, hyper-restoration; MZ, marginal zone; RCI, radial cell intercalation; RD, residual deformation; SBZ, suprablastoporal zone.

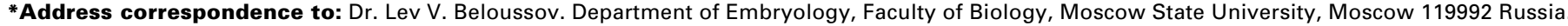
Fax: +7-495-939-4309. e-mail: Ibelous@soil.msu.ru or morphogenesis@yandex.ru
} 
Fig. 1. Biomechanical interpretation of gastrulation events in amphibian embryos. (AD, E1, E2) Biomechanical "maps" of successive stages and most important regions of embryos. Active forces are displayed by red arrows, while passive stresses are indicated by blue arrows. (A) Stretching of the blastocoel (br) roof by turgor pressure in the blastocoel. (B) $\mathrm{RCl}$ in the blastocoel roof producing pressure forces onto vegetal regions. (C) A detailed scheme of the region framed in (B). (D) Two alternating phases of biomechanical interactions between the marginal zone (MZ) and the suprablastoporal zone (SBZ). (E1, E2) Successive stages of the involution in saggital section.
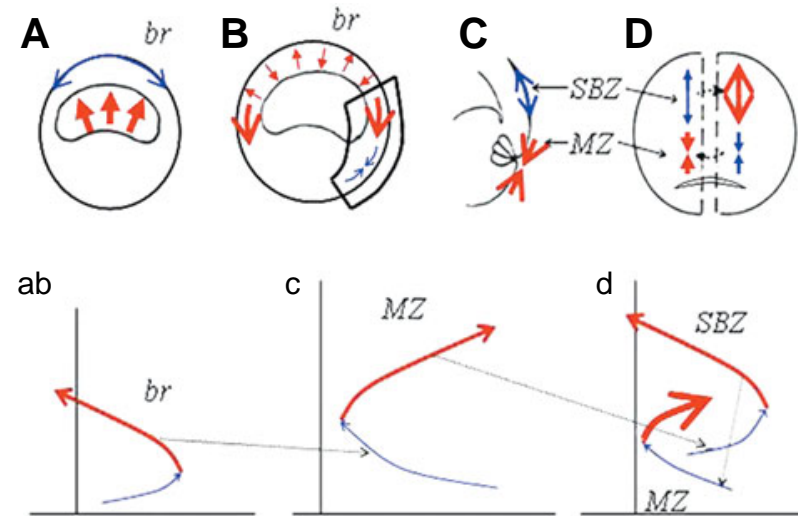
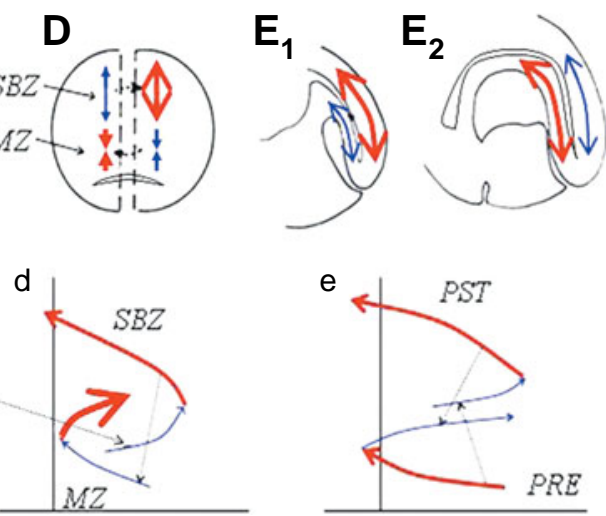

Frames (ab,c,d,e) display suggested hyper-restoration responses that correspond to the upper row frames indicated by the same capital letters (ab corresponds to $A$ and $B$ together). Dotted arrows connect the active branches of preceding hyper-restoration responses with the shifts of stress values which they produce in the neighboring parts of embryos triggering the latter's active responses. Pre, preinvoluted; pst, postinvoluted layer. gastrulation process, starting from the blastula stage, into four successive (but partly overlapping) steps regarding each of them as a single HR feedback loop creating the initial conditions for the next one. Two first steps are just preparatory, while latter two comprise the gastrulation proper. For each step, we firstly suggest a theoretical construction, then formulate some definite experimentally verified predictions and, at last, submit the supporting experimental evidence. Theoretical constructions are summarized in Fig. 1. For more detailed explanation of the terminology and schemes associated with HR hypothesis we refer the readers to the accompanying paper. Stage numbers are given by Niewkoop and Faber (1956).

\section{Step 1: Radial cell intercalation in the blastocoel roof}

\section{Theoretical interpretation}

$\mathrm{RCl}$ is a hyper-restoration reaction to the stretching of the blastocoel roof by an increased turgor pressure within a blastocoel (Fig.1 A,B; ab). As such, it obeys the contraction-extension (CE) feedback (see accompanying paper, Fig. 4).

\section{Predictions}

(1) A typical shape of amphiblastula with a thin roof and thick bottom is structurally stable and can thus be developed from a spherically symmetric state without any special initial conditions.

Suggest that the blastocoel is ideally centered and surrounded by walls of equal thickness. From our point of view, such a shape, if being pressurized from inside, will be unstable. If indeed one part of the blastocoel wall $(A)$ becomes a bit thinner than another one $(B)$ the pressure-generated tensile stress applied to $A$ will be greater than that applied to $B$ and the extension-promoted $\mathrm{RCl}$ will function predominantly in $\mathrm{A}$, leading to its further thinning. At the same time, according to $\mathrm{CE}$ feedback, $\mathrm{RCI}$ within the A wall will stimulate the tangential contraction and cell immigration in $\mathrm{B}$, making it more thick.

(2) Relaxation of the blastocoel roof should inhibit $\mathrm{RCl}$.

\section{Experimental results}

1. If one extirpates a small tissue piece from a blastocoel roof of an early - mid-gastrula (stages 10-11) embryo (Fig. 2A), within several dozens minutes it will roll into a solid ball covered by an outer ectodermal layer. Soon a cavity forms inside, whose spherical shape indicates that it is under turgor pressure. The cavity gradually shifts out of the central position, one of its walls becoming very thin while the opposite one becomes thick and indented, indicating cell immigration (Fig. 2 BD; see for more details Beloussov and Petrov, 1983). This shows that $\mathrm{RCl}$-mediated eccentricity of a blastula cavity is a structurally stable property, not associated with some unique initial conditions.

2. If one relaxes circumferential tensions at the blastula stage (9-10) by inserting a wedge of endodermal tissue into a vegetal hemisphere of an embryo (Fig. 3A), the process opposite to $\mathrm{RCI}$ is taking place, namely the clustering of deep cells of the blastocoel roof into a condensed ensemble (Fig. 3 B,G). Additionally, several hours after the operation the animal surface of relaxed embryos becomes ruffled and acquires several indentations, later on taking the shape of large folds and protrusions (Fig. 3C). Obviously, all these events promote a circumferential contraction of the blastula surface and hence an increase of tensions on its surface. For example, the formation of just a single protrusion (like that shown in Fig. 3C) stretches the surrounding surface area for no less than about $15 \%$ and usually more than one protrusion is created. As a rule, such embryos do not exhibit gastrulationassociated involution movements. The axial organs, if formed, are arranged quite abnormally (Ermakov and Beloussov, 1998; Beloussov and Ermakov, 2001). Meanwhile, if we restore the
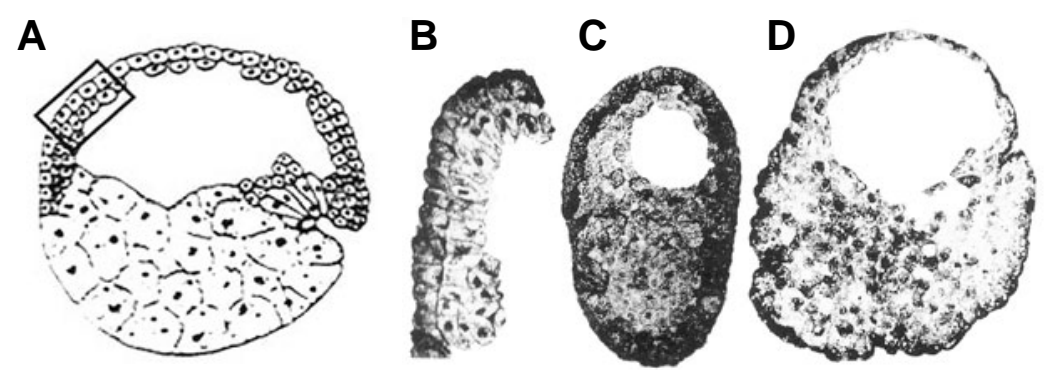

Fig. 2. Formation of a very much diminished copy of a normal amphiblastula from a ventral ectoderm explant of the early gastrula $X$. laevis embryo. (A) Scheme of operation. (B-D) An explant immediately after extirpation, 8 and $24 \mathrm{~h}$ later. From Beloussov and Petrov, 1983. 
tensile stresses soon after their relaxation (either by putting embryos in hypotonic solution which increases the water flow into the blastocoel or by direct embryos stretching with the use of two needles) the $\mathrm{RCl}$ is renewed and a subsequent development normalized (see Fig. 3 D, G, and E,H,F). The corresponding quantitative data are summarized in Fig. 31 (for more details see Beloussov and Ermakov, 2001).

\section{Step 2: Formation of bottle-shaped cells in the marginal zone}

\section{Theoretical interpretation}

This is the HR reaction to this zone relaxation/ compression by the actively extended blastocoel roof (Fig. $1 \mathrm{~B}, \mathrm{C}, \mathrm{ab}, \mathrm{c}$ ).

\section{Predictions}

(1) Bottle-shaped cells should actively contract themselves in the direction of their relaxation/compression by an actively extended blastocoel roof (that is, in the dorso-ventral direction).

(2) Artificial relaxation of embryonic tissue can induce the formation of bottle-shaped cells in unusual locations.

\section{Experimental results}

1. The existence of tangential pressure exerted by the blastocoel roof towards more vegetal embryo regions is confirmed by planimetric measurements performed at the late blastula stage (Keller, 1978): the square of the blastocoel roof is significantly increased within this period, while that of the $M Z$ remains constant. The expected dorso-ventral (meridian) contraction and hence transversal (latitudinal) flattening of bottle-shaped cells has been directly observed (Hardin and Keller, 1989). The authors found such a highly anisotropic contraction not to be an intrinsic property of bottle-shaped cells, because in isolated explants they contract isotropically.

2. The excessive bundles of bottle-shaped cells are produced in the following abnormal locations as a consequence of tissue relaxation:

(a) onto the SBZ surface relaxed at stages $10-11$ by $P$-shaped incision and separation from underlain tissue (Fig. 4A; see for details Beloussov, 1988);

(b) on the bottom of a newly arisen pit formed onto the tip of a relaxed at stages $10^{1 / 2}-11$ dorsal blastoporal lip (Fig. 4 B-D). The lip was firstly isolated and then relaxed by a longitudinal incision (Fig. 4B). Its reaction to the relaxation consisted of two successive steps. The first one (Fig. 4C) is passive. It takes no more than a few seconds and can proceed under low temperatures $\left(4-60^{\circ} \mathrm{C}\right.$ for Rana temporaria and $10-$ $120^{\circ} \mathrm{C}$ for Xenopus laevis embryos). The second one (Fig. 4D) is active (occuring only under normal temperature) and takes several dozen minutes. As a result, the lip's curvature is inverted (cf Fig. $4 \mathrm{~B}, \mathrm{D})$. This is a clear overshoot reaction, because for restoring tensions without an overshoot it would be enough for a dissected lip to return to the curvature of the blastula surface.
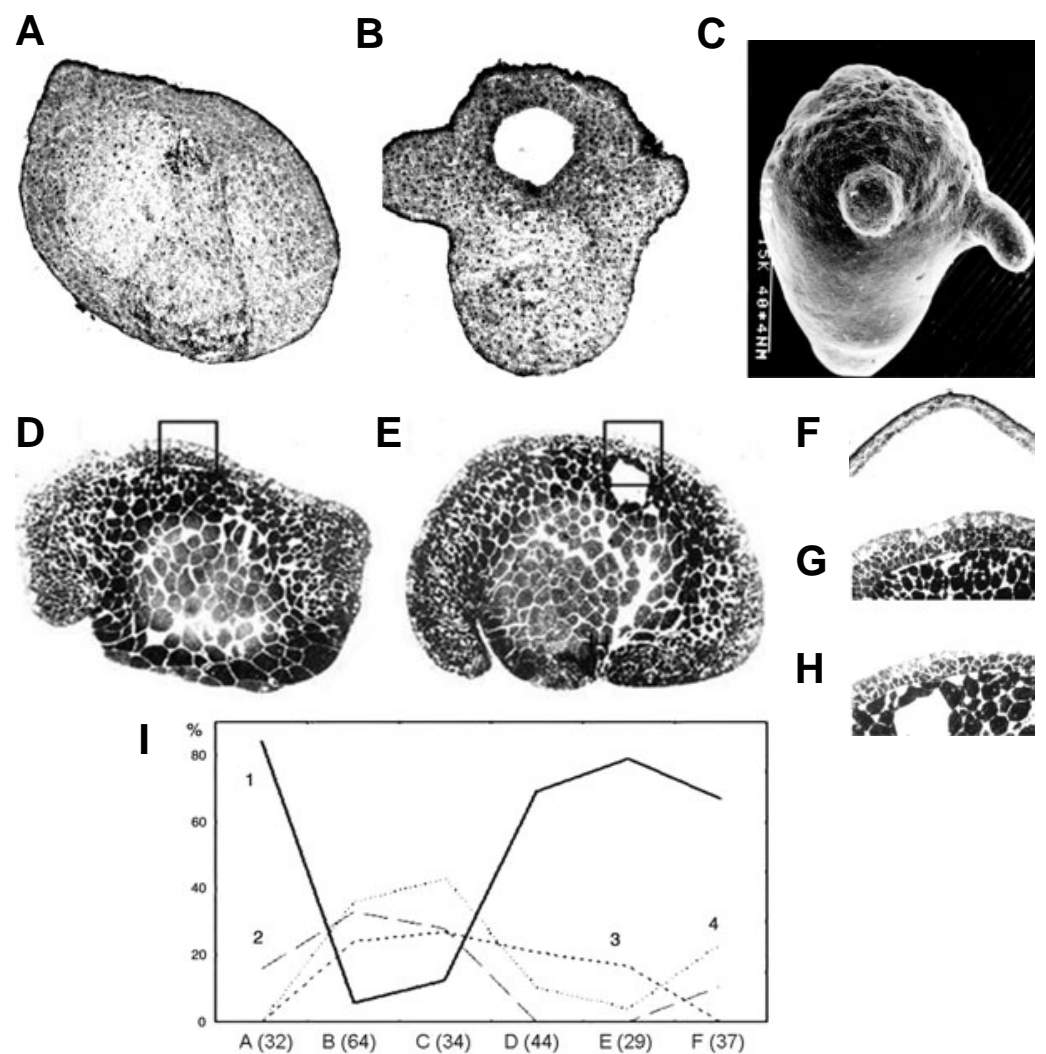

Fig. 3. Results of relaxation of tensile stresses at the surface of the late blastula stage of $\boldsymbol{X}$. laevis embryos. (A) An embryo with a wedge-shaped implant causing relaxation, $1 \mathrm{~h}$ after operation. (B) A similar embryo, $5 \mathrm{~h}$ after operation. (C) Scanning electron microscopy view of a relaxed embryo, 1 day after operation. Note two extensive protrusions which stretch the surrounding tissue areas. (D) A saggital semithin section of a relaxed embryo maintained for $5 \mathrm{~h}$ in $50 \%$ MMR solution. (E) $A$ similarly treated embryo maintained for the same time period in 25-fold diluted MMR, tensions being considerably restored. (F) Fragment of the blastocoel roof of an intact embryo of the same age as $(B)$. $(\mathbf{G}, \mathbf{H})$ Similar fragments from $(D, E)$ embryos, respectively. Note the reduction of the number of cell layers in (F) as compared with $(B)$ and in $(H)$ as compared with $(G)$, indicating the renewal of $R C l$ after incubation in hypotonic solution. (I) A diagram summarizing the results of "stress therapy" of the relaxed embryos. (A) Control group; (B) relaxation by a frontally oriented radial cut through a vegetal embryo region; (C) a similar relaxation by a saggitally oriented radial cut; (D) embryos incubated in a hypotonical (25-fold diluted) MMR after frontally oriented cut; (E) similarly treated embryos after saggitally oriented cut; (F) frontally oriented cut followed by stretching of the embryos. Figures in brackets give the number of samples in the corresponding series. Vertical axis, percent of normalized and abnormal embryos $24 \mathrm{~h}$ after operations. Graph 1, normal embryos; 2, exogastrulation; 3, incomplete gastrulation; 4, other anomalies. Note a substantial recovery after tension renewal. From Beloussov and Ermakov (2001).

(c) on the ventral embryo surface after relaxation of circumferential tensions at the early gastrula stage $\left(10-10^{1 / 2}\right)$ by a dorsomedial cut (see below for more details).

\section{Step 3: Gastrulation proper; epiboly and involution}

\section{Theoretical interpretation}

We suggest that the main driving force of gastrulation is the contraction-extension (CE) feedback established between $\mathrm{MZ}$ and the surrounding embryonic surface, first of all between the dorsal blastoporal lip and SBZ (Fig. 1 C,D,d). This feedback is 


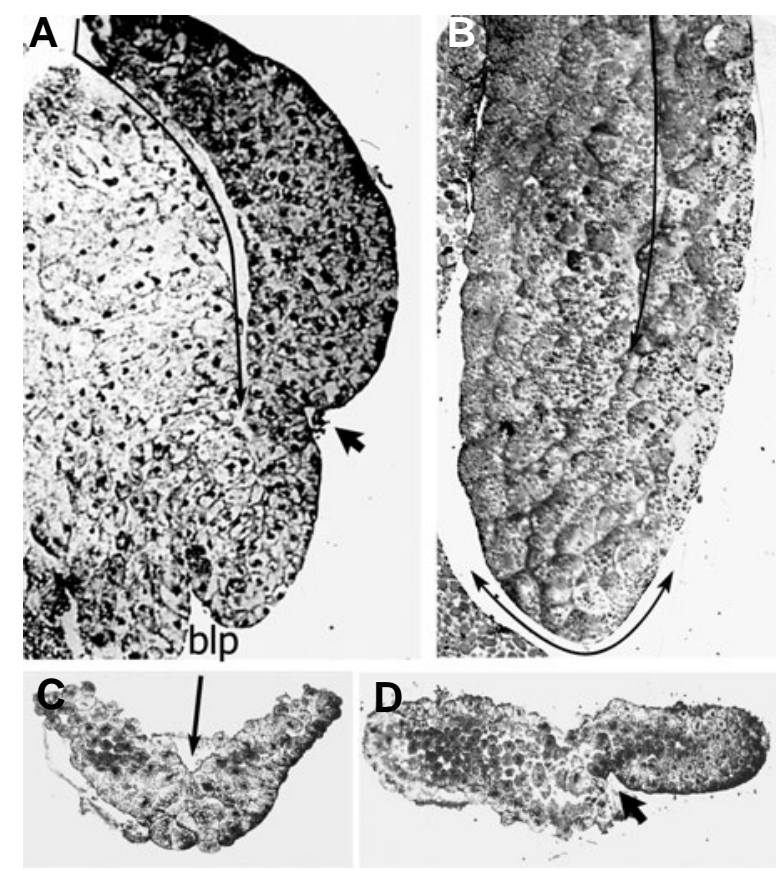

A

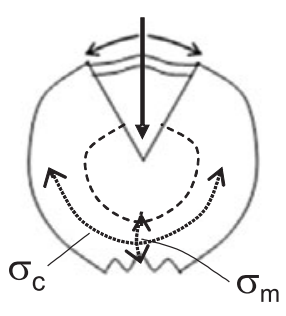

B

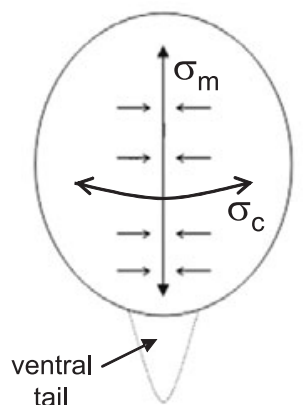

tail

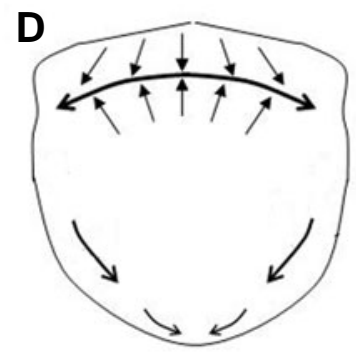

Fig. 4 (Left). Relaxation-promoted formation of additional groups of bottle-shaped cells and the corresponding invaginations (shown in A, D by dense pointers) (A) $1 \mathrm{~h}$ after the relaxation of the suprablastoporal zone due to its separation from the underlain tissues (large bent arrow) at the early gastrula stage. blp is the normal blastopore. (B) A saggital section of the dorsal blastoporal lip of the normal mid-gastrula stage embryo. Arrows show separation directions which relax pre-existed tensions. (C) A relaxed lip immediately after separation (D) $1 \mathrm{~h}$ later, a new invagination pit has been formed at the site of a former dorsal blastoporal lip (pointer).

Fig. 5 (Right). Schemes of the operations for modulating $\sigma_{c}$ and/or $\sigma_{m}$ values (shown by dotted lines) in the marginal zone of early gastrula embryos and expected results. (A) A transversal view of a dorso-medial cut (vertical arrow in A) providing wound opening (diverged arrows) and hence $\sigma_{c}$ relaxation, most of all in the ventral region (wavy part of a contour). Wound gap is covered by a piece of ventral ectoderm. (B) Expected cell convergence along a ventral mid-line due to increase of inequality between a preserved $\sigma_{m}$ (vertical bidirectional arrow) and relaxed $\sigma_{c}$ (convex arrow). View from the ventral side. (C) Enhancement of $\sigma_{c}$ in the dorso-medial zone as a result of transversal stretching by two needles (arrows coming from black spots). (D) Expected result; an extensive transversal cell convergence in the area of the suprablastoporal zone and latero-ventral cell movements due to some $\sigma_{c}$ relaxation in the ventral region.

initiated by the above mentioned meridian contraction of the bottle-shaped cells which triggers the active (convergent) extension of SBZ. This latter in its turn relaxes/compresses the MZ, promoting its contraction and so on. As a result, $M Z$ corresponds to the $\alpha$ zone, while SBZ corresponds to the $\beta$ zone (see Fig. 4 from Beloussov and Grabovsky paper, this volume). As gastrulation proceeds, CE feedback extends to more vegetal $\mathrm{MZ}$ regions. The involution of cell material around the blastoporal lip is a clear indication of an overshoot SBZ response because the linear rate of the active SBZ extension exceeds that of the MZ contraction (such an excess has been directly demonstrated by time-lapse filming: Glagoleva et al., 2003). At the same time, the blastoporal lip, while becoming a part of a toroidal surface, starts to work as a funnel (see Fig. 3D from Beloussov and Grabovsky paper, this volume), converging the adjacent cells towards the dorsal embryo midline.

\section{Predictions}

1.The location of the dominating meridian of cell convergence as well as the direction of convergence should depend upon the tensile pattern within the $\mathrm{MZ}$, rather than being an autonomous property of converging cells.

As argued by Beloussov and Grabovsky (this volume), the MZ is a semi-toroidal surface pressurized from inside, with its merid- ian tensions $\left(\sigma_{\mathrm{m}}\right)$ approximately twice as high as the latitudinal (circumferential) ones $\left(\sigma_{c}\right)$. According to the HR hypothesis, the inequality $\mathrm{D} \sigma=\left(\sigma_{\mathrm{m}}-\sigma_{\mathrm{c}}\right)>0$ promotes cell convergence towards meridians and hence a circumferential contraction of the MZ, which normally leads to the blastopore closure. By varying this inequality in different MZ locations, we expect to modify in a predictable way the rate and the direction of cell convergence. The following interventions of such a kind may be suggested:

(a) To relax tensions by a dorso-medial cut, directed towards a center of embryonic body (Fig. 5A). This operation should diminish $\sigma_{c}$ throughout an entire $M Z$ and also $\sigma_{m}$ in the cut vicinity, but not opposite to it, that is, along the embryo's ventro-medial meridian. Correspondingly, in the latter region $D \sigma$ will take the highest value and hence cell convergence should be expected to go towards the ventral, rather than dorsal midline, that is opposite to its normal direction (Fig. 5B).

(b) To make the same operation, but in addition to relax $\sigma_{\mathrm{m}}$ along the entire $M Z$ by isolating it from the rest of embryo. Under these conditions $D \sigma$ in the ventro-medial region will not be increased and hence no ventralwards inversion of convergent movements should be expected.

(c) To increase $\sigma_{c}$ in the SBZ by stretching this area transversely (Fig. $5 \mathrm{C}$ ). We expect that under extensive stretching $\sigma_{\mathrm{c}}$ will exceed $\sigma_{m}$, enforcing cell convergence to go along the 
transversal axis, that is, perpendicularly to its normal direction. At the same time, such a stretching should relax $\sigma_{c}$ in the lateroventral $\mathrm{MZ}$ regions and hence promote ventralwards cell convergence as in the previously suggested experiment (Fig. 5D).

2. Cell convergence towards meridians should take place in any internally pressurized fold (even if prepared artificially), or in a tissue "scroll", irrespective of its specificity.

\section{Experimental results}

1 (a). In X. laevis embryos destined for the operations, at 32 blastomeres stage two ventral (Fig. 6 A-C) or two dorsal (Fig. 6D) blastomeres were labeled by microinjecting fluorescein-dextran. At the early gastrula stage $\left(10-10^{1 / 2}\right)$ a dorso-medial cut was made through the SBZ and the dorsal blastoporal lip. The wound gap was covered by a piece of ventral ectoderm in order to weaken wound healing reactions at the gap edges. In the embryos with ventral tissue labeled, within a few hours after operation, condensation of the labeled material along the ventral embryo midline was observed. Later on an abnormal tail formed on the ventral (rather than dorsal, as normally) lip of the blastopore (Fig. 6B). This was in a sharp contrast with the same cells movements in unoperated embryos, which are always directed dorsally (Fig. 6A). Correspondingly, due to the same operation the labeled dorsal material also moved ventralwards and formed stripes aligned along the lateral lips of the blastopore (Fig. 6D), which is strictly opposite to the normal dorsalwards convergent movements. Hence, according to our expectations, a complete inversion of cell convergence movements took place in these experiments.

1(b). Before making a similar dorso-medial cut, we decreased $\sigma_{m}$ by cutting off an entire $M Z$ from the rest of embryo. In these, "sausage-like" MZ preparations no ventralwards convergence took place at all (Fig. 6D). Instead, a patch of labeled cells has extended towards the edges of the explant.

1 (c). We first give an overall view of the movements of the labeled cells and then show the morphological results of transversal SBZ stretching.

As previously, two dorso-medial blastomeres were labeled at
A

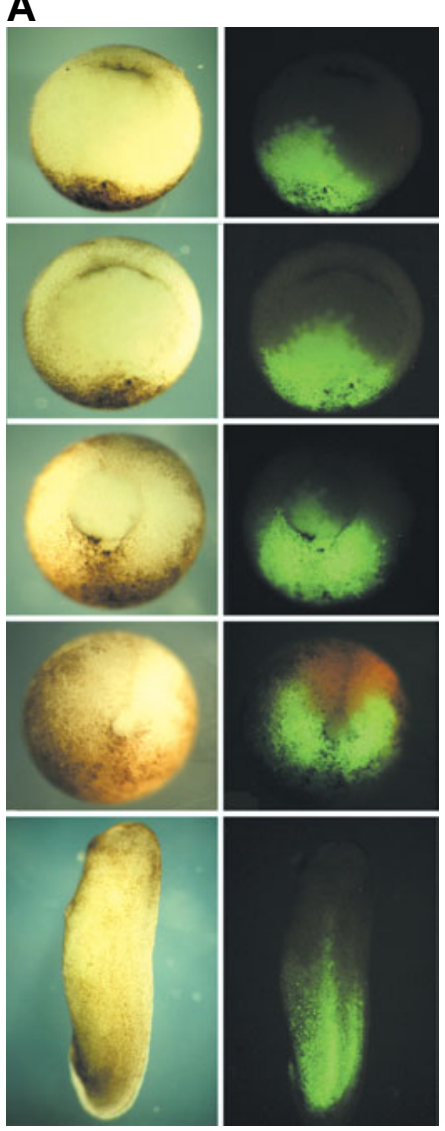

B
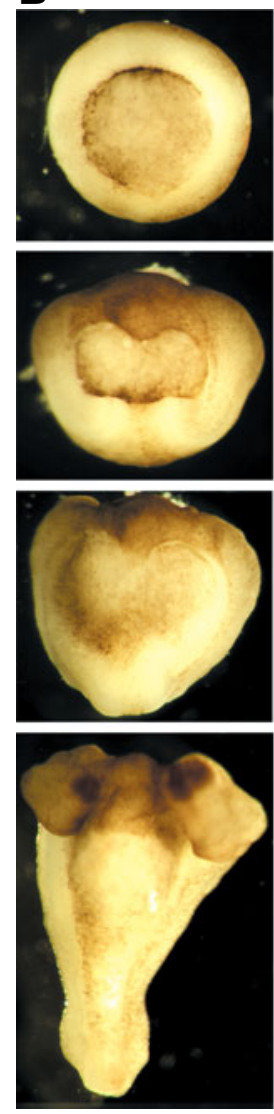
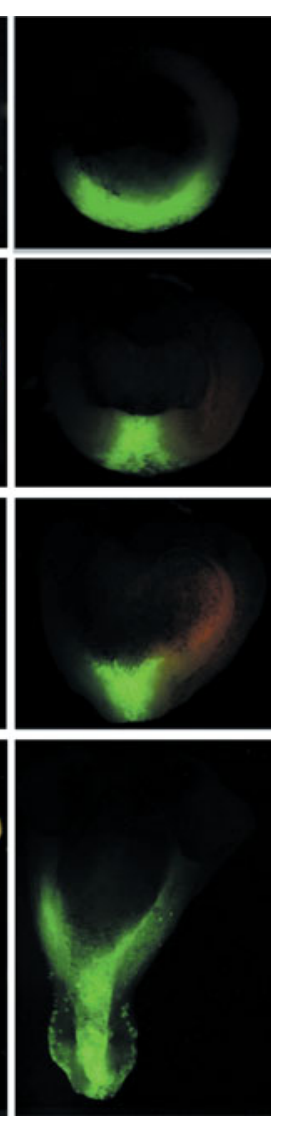

C
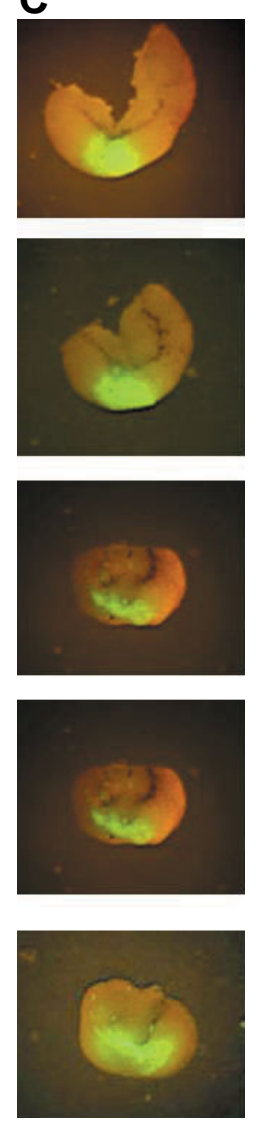

D

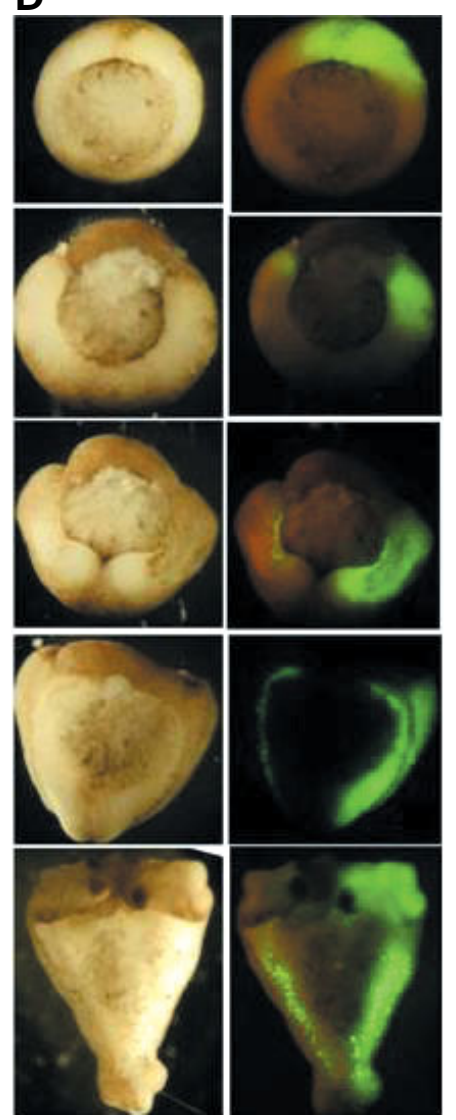

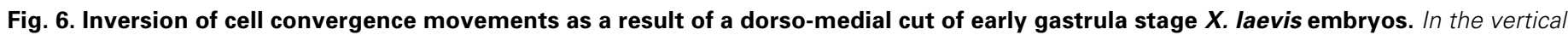

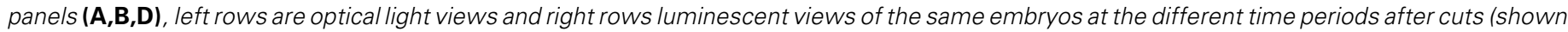

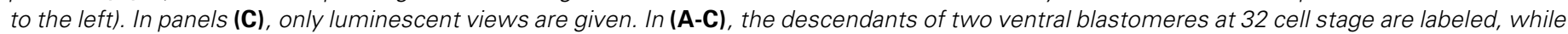

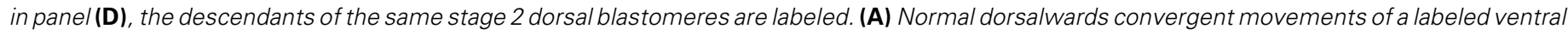

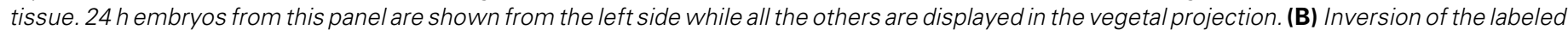

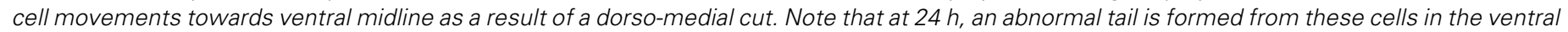

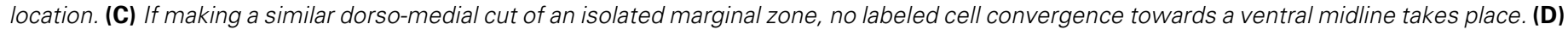

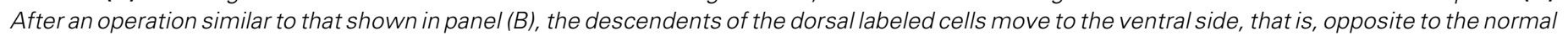
convergence direction. As a result, they spread along the lateral lips of the blastopore. 

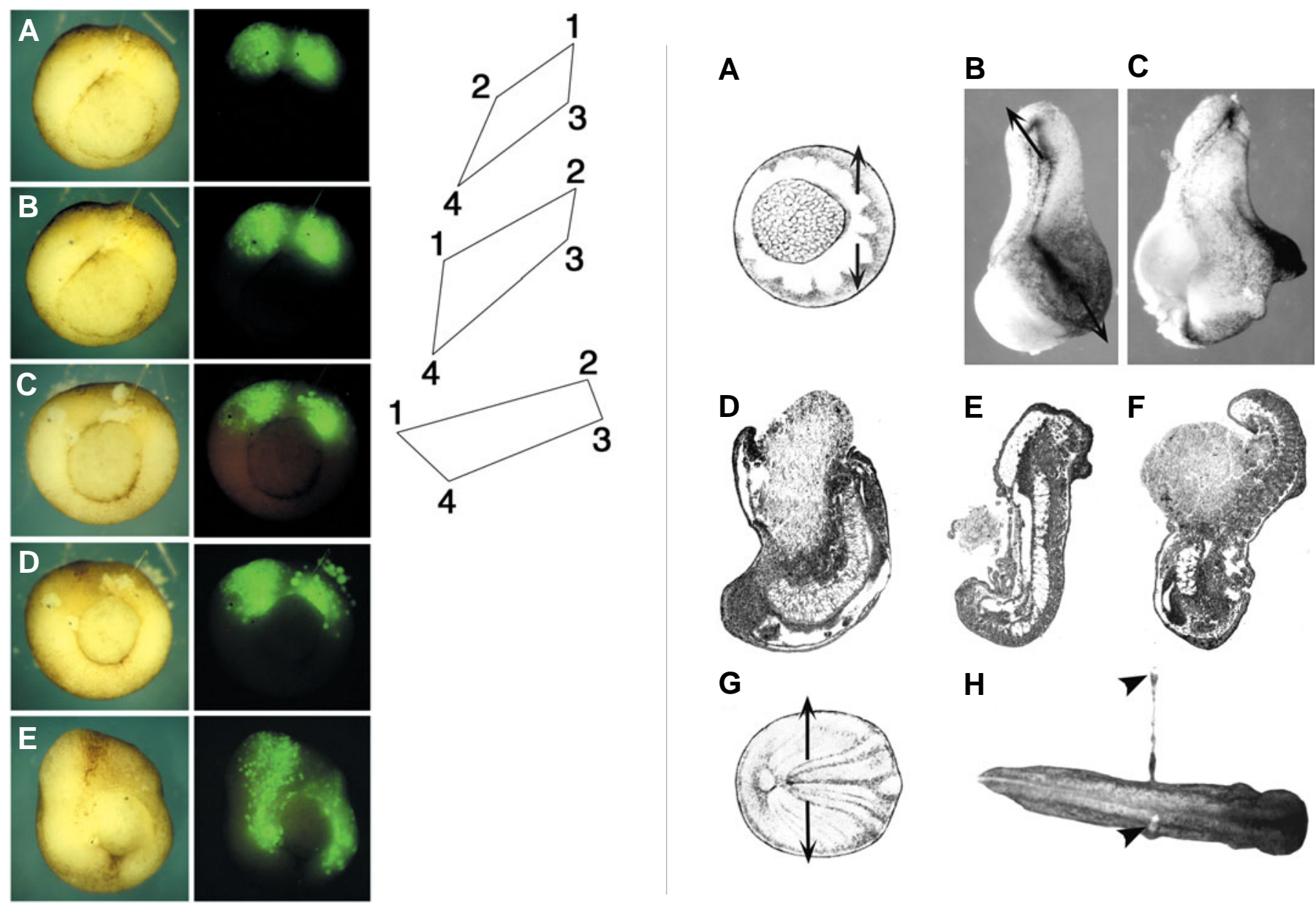

Fig. 7 (Left). Induction of the active lateralwards movements of the labeled descendants of dorsal cells as a result of transversal suprablastoporal zone stretching by four needles. To the left: optical light and luminescent views just after inserting needles and before stretching them (A), immediately after stretch (B) at $2.5 \mathrm{~h}$ (C), $3.5 \mathrm{~h}$ (D) and $7 \mathrm{~h}$ (E). In optical light views, needles are denoted by black spots. To the right are shown the positions of the needles 1-4 before stretching, immediately after stretch and $2.5 \mathrm{~h}$ later. Note a considerable post-stretching increase of the distance 1-2 in spite of the needles being anchored to the agarose substrate. As a result, the dorsal cell material is very much shifted to the ventral, considerably overlapping the needles' positions. This result is quite similar to that obtained by a dorso-medial cut (see Fig. 6).

Fig. 8 (Right). Morphological results of suprablastoporal zone (SBZ) transversal stretching, as compared with a similar stretch of neurula stage embryos. (A) Scheme of SBZ stretching by two needles. (B-F) $24 \mathrm{~h}$ results. (B,C) Total views (on frame B, the direction of stretching is indicated), whereas (D-F) are histological sections made in transversal embryo planes. Note a complete transversal reorientation of the embryo body including not only the axial rudiments (notochord and somite series), but also the yolk-containing compartments. In (D) and to some extent in (F) part of the notochord is shifted to the lateral blastoporal lip. (G) A scheme of a similar operation performed at the early neurula stage. (H) Its result, total view. Contrary to $(A-F)$, the larva fully preserves its initial axis, remaining bound to one of the needles by a thin unstretched tissue thread (later on disrupted). In (H), pointers indicate the positions of the needles.

the 32 cells stage. When the embryos reached early gastrula stage, the SBZ was stretched by use of 4 needles (Fig. 7 A,B). By 2,5 h after fixing the needles in the stretched positions a ventralward movement of the labeled cell material could be traced. It was so extensive that the tip of needle \#1 was involved, in spite of its lower part being anchored in the agarose substrate (Fig. 7 B,C). Later on this movement was continued, leading in $7 \mathrm{~h}$ to shift of the labeled cells towards the lateral lips of the blastopore, that is, greatly overlapping the needles' shifts (Fig. 7 D,E). Therefore, active stretch-induced cell movement quite similar to that induced in the previous experiments could be observed.

If the SBZ undergoes two rounds of stretching (being stretched in the total for no less than about $120 \%$ of its initial length) and maintained stretched for about $20 \mathrm{~h}$, a reorientation in the stretch direction of entire embryo body, including not only the axial organs (notochord, neural tube and somite series), but also their vegetal parts, takes place (Fig. 8 A-D). We observed this result in 10 cases out of 15 (Fig. 8 A-D). In some cases (Fig. 8 D,F) a part of the notochord shifted towards the lateral blastoporal lip indicating the same ventralwards movements as previously described.

It is of interest to compare these results with those obtained by a similar transversal stretching of an early neurula (stages 13-14) embryo (Fig. 8G). In the latter cases even extensive stretching could not force the longitudinal embryo axis to be reoriented. The 
only result of such manipulation was the extrusion of a thin tissue thread (later on disrupted) towards one of the needles (Fig. $8 \mathrm{H}$ ). Therefore, the capacity for a profound stretch-dependent reorientation of cell movements was a property of gastrulation stage embryos only. Later on the stage-specificity of biomechanical cell reactions will be discussed in more detail.

2. Cell convergence in the artificially prepared folds and tissue "scrolls".

If it is true that the very geometry of a fold provides cell convergence towards its midline, this effect should take place not only on the "natural", but also on the artificially prepared folds. To check this hypothesis, we extirpated from a midgastrula embryo (stage $11-11^{1 / 2}$ )a piece of ectoderm situated laterally to the dorsal midline, folded it by its outer surface outside and fixed the fold with a glass needle (Fig. 9A). At about $20 \mathrm{~h}$ after operation we found, in 4 cases out of 6 , rather long protrusions that had grown from a midline of the folded surface (Fig. 9 B,C). The formation of protrusions indicates cell convergence going towards the midline meridian of a bent surface. Such an effect took place only with gastrula stage embryos and not later. However, at advanced stages $(12-17)$ a similar reaction could be observed on dissected pieces of the lateral ectoderm (Fig. 9D) which after isolation spontaneously rolled down into scrolls, the latter's long axes coinciding with those of the entire embryos. When observing these formations after several dozens minutes, we could easily trace an extensive longitudinal contraction and formation of a series of transversal grooves and ribs (Fig. $9 \mathrm{E}-\mathrm{G}$ ). This indicates cell convergence towards several more or less arbitrarily located meridians of a scroll. These experiments show that the process of meridian cell convergence is rather nonspecific.

3. Relaxation rates of tensile and compression stresses and their relations to the rates of gastrulation movements.

To maintain CE feedback (Beloussov and Grabovsky, this volume), embryonic tissues should be tensed. This will be possible only if the time required for the relaxed/compressed sample for at least restoring (if not hyper-restoring) its initial tension due to its active contraction (let us denote this time as $T_{a c}$ ) is much smaller than the time required for a passively extended tissue area to develop the active intercalation-promoted pressure $\left(T_{i p p}\right)$ : in the opposite case the entire system will be pressurized, rather than tensed. On the other hand, the reverse $T_{\text {ipp }}$ (i.e., the rate of the active pressure-generated extension) should not be too small in comparison with the rate of the epibolic tissue movements
A

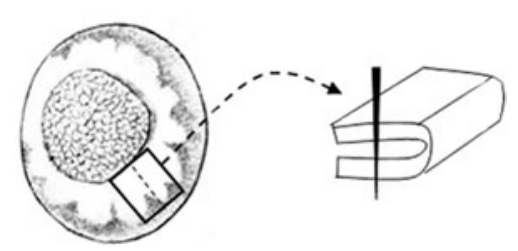

B

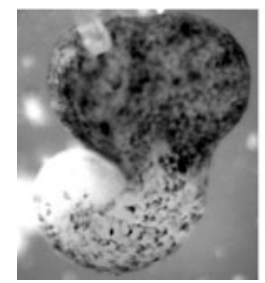

C

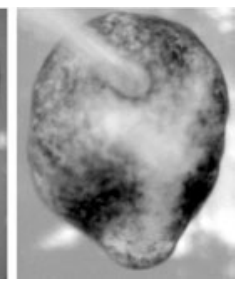

D

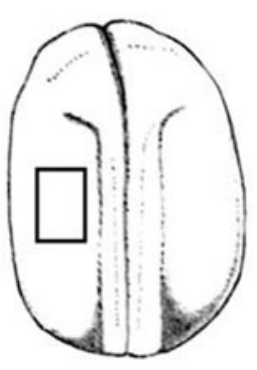

E

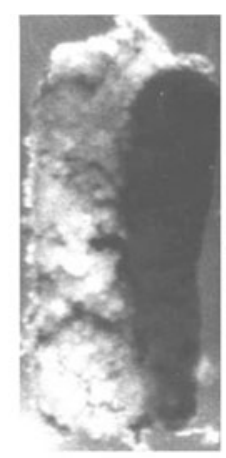

$\mathbf{F}$

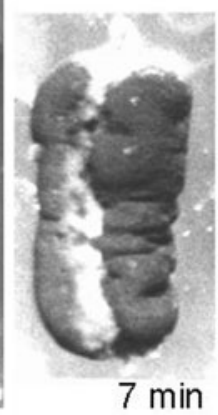

G

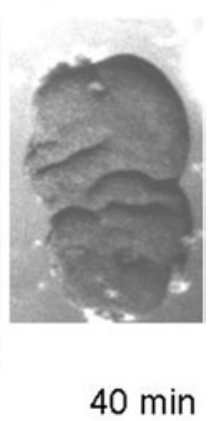

A
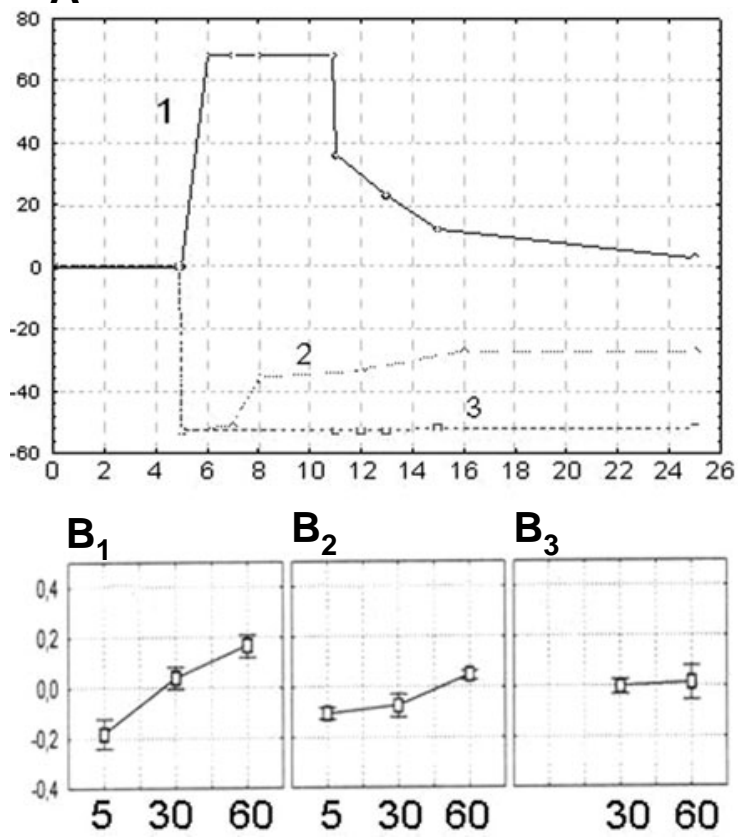

Fig. 9 (Left). Evidence of cell convergence on artificial folds and ectodermal "scrolls". (A) A scheme of fold preparation; an ectodermal tissue piece situated lateral to the dorsal mid-line of a gastrula stage embryo is cut off, bent as shown by a dashed line and fixed in this position by a needle. (B,C) Protrusions formed in 10-15 h out of the bent folds (opposite to the needles). (D) If a framed region of lateral ectoderm is extirpated from the neurula stage embryo, it rolls spontaneously into a scroll, its longitudinal axis coinciding with that of embryo (E). Soon the scroll is transformed into a meridionally shrunk body with many transversal grooves and ribs (F,G). Times after scroll formation are shown.

Fig. 10 (Right). Residual deformations (RDs) in stretched or shrunk explants of embryonic tissues after their unloading. Vertical axes: percents of the positive (fixed elongation) or negative (fixed shrinkage) RDs. Initial length is taken as zero. Horizontal axes: time, minutes. (A) Protocol graphs of a stretched explant (1) and two shrunk explants (2,3). Explant 1, stretched to $70 \%$ at 5 min time and unloaded 6 min later, gradually returned to its initial length. Meanwhile, explant 2 which was shrunk to $50 \%$ and kept in this state for only 2 min gave - 35\% RD; explant 3 shrunk for 5 min and gave - $100 \%$ $R D$. (B) RD after unloading 10-20\% stretch in 5, 30 and 60 min after its application. $\left.\mathbf{( B}_{\mathbf{1}}\right)$ Explants of the early gastrula ventral ectoderm, $\left(\mathbf{B}_{\mathbf{2}}\right)$ SBZ tissue, $\left(\mathbf{B}_{3}\right)$ explants of the lateral ectoderm from neurula stage embryos. While in 5 min after force application the samples $B_{1}$ and $B_{2}$ undergo $20-10 \%$ contraction, (that is, negative $R D$ ), after 60 min stretch, $B_{1}$ samples gave $+20 \% R D, B_{2}$ samples just a slight $R D$ and $B_{3}$ samples no $R D$ at all. 
during gastrulation, because otherwise the gastrulation movements would be hampered by increased tensions.

We tested $\mathrm{T}_{\mathrm{ac}}$ and $\mathrm{T}_{\mathrm{ipp}}$ by shrinking or stretching the sandwich explants of the ventral ectoderm and SBZ taken from $10^{1 / 2}-11^{1 /}$

2 stage embryos during different time periods and to different extents. The residual deformations (RD) after unloading have been measured (see Glagoleva et al., 2003, for more details). Some of explants were marked by carbon particles for testing cell movements either in a shrunk or in a stretched state. It turned out, that the time of stabilization of a shrunk state did not exceed $5 \mathrm{~min}$ and in about 10 min after shrinkage the active contraction movements started. Therefore, $\mathrm{T}_{\mathrm{ac}}$ did not exceed $10 \mathrm{~min}$. On the contrary, $\mathrm{T}_{\text {ipp }}$ was no less than $30 \mathrm{~min}$. If relieved earlier, a stretched explant contracted up to its unstretched state (Fig. 10A). A substantial RD could be traced only in 60 min after imposing a $10-20 \%$ stretch (Fig. $10 \mathrm{~B}_{1}$ ). It is noteworthy, under the same amount of deformation and stretching time the RD of SBZ samples were very small (Fig. $10 \mathrm{~B}_{2}$ ), reaching $20 \%$ only after 40 $80 \%$ stretch (not shown). Hence, the rate of relaxation of moderate stretching stresses in the ventral ectoderm was greater than in the SBZ. Obviously, during epiboly this should promote a dorsomedial shift of embryonic tissue from the rapidly to slowly relaxed area, that is from the ventral towards dorsal. This is just what is normally observed.

Now let us compare the rate of the linear extension of an animal embryo hemisphere during epiboly with the rate of tension relaxation in the same tissue. The first value, measured just between stages $10^{1 / 2}$ and $11^{1 / 2}$ was found to be from 8 to $12 \%$ per hour (Keller, 1978). Meanwhile, the explants of the blastocoel
A

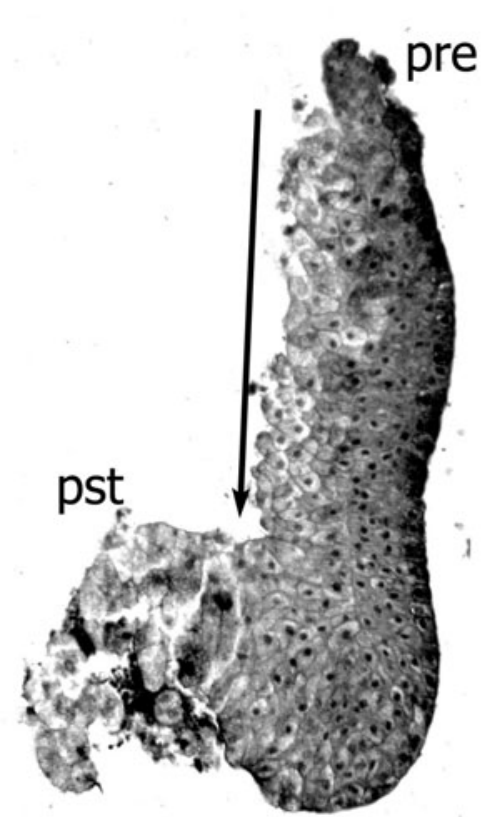

B

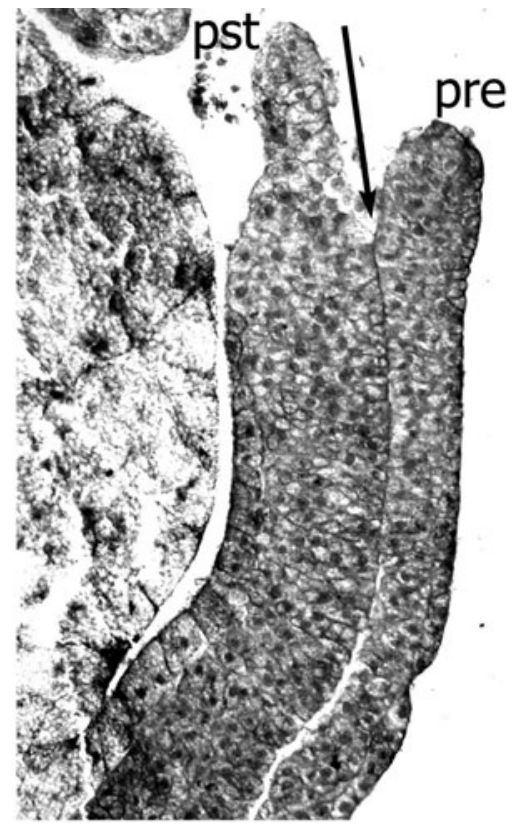

Fig. 11. Exchange of the passive stretching of the postinvoluted layer (pst) at the early-mid gastrula stage (A) by its active extension (B). Arrows show the directions of the separation of the pre-involuted (pre) and postinvoluted layers. Samples were fixed in Bowen's fluid a few seconds after separation and sectioned saggitally. (A) An extensive contraction and curling of pst. (B) Its active extension, overlapping the layer pre. In the both frames the anteroposterior direction goes from up to down. roof, stretched for an hour up to $20 \%$ of their initial length retained, after stretch relief, just $10 \%$ of their initial length. Consequently, the rates of normal gastrulation movements roughly satisfy the condition of constant tension. One may conclude that the rate of tension relaxation is the limiting parameter of gastrulation movements.

On the other hand, the lateral ectoderm explants taken from early neurula embryos (stages 13-15) did not show RD at all after any amount and duration of stretch (Fig. $10 \mathrm{~B}_{3}$ ). Hence, at these stages the imposed tensions are not relaxed at all. One concludes that the ability to relax tensions by cell intercalation is a stagespecific property of gastrula stage embryos only.

\section{Step 4: Extension of the postinvoluted cell material}

\section{Theoretical interpretation}

EE feedback (see Beloussov and Grabovsky, this volume) is expected to be established between preinvoluted (pre) and postinvoluted (pst) cell material. Namely, at the beginning of involution $p s t$ is assumed to be passively extended by the actively extending pre, while later on pst becomes an active component of the mutually promoting EE feedback (see Fig. $1 E_{1}, E_{2}, e$ ).

\section{Predictions and experiments}

While carefully detaching the adjacent preand pst areas from each other, one should expect that at the start of involution pst will be immediately and extensively contracted (relieving the passive tension), while at the later stages it can even overlap the overlain pre. Just this was observed: if we made such a detachment at stage 11 (mid-gastrula), an extensive and immediate pst contraction and curling took place (Fig. 11A). Meanwhile, at stages 13-14 the pst, instead of being contracted, in few seconds extended, overlapping pre (Fig. 11B).

\section{Discussion}

The main goal of this study was to show that the entire process of amphibian gastrulation can be represented as a succession of mutual biomechanical reactions between the different parts of an embryo, the earlier reactions creating the initial conditions for the next ones. In this account, for the sake of brevity, several points have been omitted or just briefly discussed. Among those is the latero-ventral circular spreading of a blastoporal slit and some detail of involution. The adequate biomechanical interpretation of these events is given by Cherdantzev et al. (this volume).

We do not know any experiments or the observations of a normal gastrulation process which, after a proper analysis, contradicts our interpretation. This includes, among others, the detailed description of morphogenetic events within the isolated MZ (Davidson etal., 2002). Moreover, we believe that our interpretation can be applied to other types of gastrulation as well, at least in the case of holoblastic embryos. In all these cases, the crucial point is the establishment of CE feedback in the blastocoel walls: one (animal) part of a spherical wall becomes actively extended while another one (vegetal) becomes actively contracted. If the blastula wall includes more than one cell layer in width, the animal extension is achieved by $\mathrm{RCl}$ while in one cell 
layered blastulae (as in sea urchins) the same should be achieved by cell flattening. On the other hand, the vegetal contraction can proceed with participation of either cell immigration, or tangential cell contraction, or both together. The above described experiments (Fig. 2 and the corresponding comments) show that a segregation of a blastula wall into extended and contracted parts can go in the absence of any pre-established polarity, although normally it coincides with the earlier positioned animal/vegetal egg axis.

At a next step, the contraction of the vegetal surface will make it flatter than the animal one and so the both zones will be separated by a circle of an increased curvature which is the rudiment of a blastoporal lip. Hence taking the shape of a semi-toroidal surface, the lip should start to contract circumferentially, thus leading to the blastopore closure. Such may be a rough sketch of the processes involved in all kinds of gastrulation.

What is the relationship of our approach to research directed towards deciphering the molecular machinery of gastrulation? In our view, both approaches are complementary (see also Keller et al., 2003), because the active responses to mechanical stresses require a refined molecular machinery, whose disturbances should largely modify the rates and other parameters of the standard morphogenetic responses. Detailed studies of these effects are in progress in our research group. On the other hand, certain elements of the genes expression patterns should be considered as an initial condition for the entire morphomechanical causal chain. This relates first of all to the establishment of dorso-ventrality, which takes place long before the beginning of gastrulation (Gerhart and Keller, 1986). It is noteworthy, that this process is not purely chemical, but includes also mechanical events, namely the rotation of the egg cortex and/or yolk flows (see Cherdantzeva and Cherdantzev, this volume). It is easy to see that these mechanical forces are directed towards the relaxation/compression of the future dorsal side of an egg, as related to its ventral part. Taking into consideration that by Farge (2003) data it is pressure that initiates the migration of $\beta$-catenin to cell nuclei of Drosophila embryonic tissues, one may speculate that similar processes involved in dorsalization of amphibian embryos (Schneider et al., 1996) may be triggered by the same mechanical impulse.

Therefore we suggest that the relationships between the morphomechanics and molecular machinery are bi-directional: a molecular machinery affects mechanics and vice versa. Such a view fits the approaches, emphasizing the role of cell movements (Yamada,1994; Salazar-Ciudad et al., 2003) or mechanical waves (Gordon, 1999) in affecting cell differentiation, because the both kinds of events are associated with mechanical stresses. Our results presented above permit us to formulate some definite questions addressed directly to the molecular level. For example, one should ask whether $\mathrm{CCI}$ movements taking place, after remodeling of mechanical stresses, in quite abnormal directions and locations are associated with the activation of the same signal pathways as the "standard" convergent movements. The corresponding investigations are now in progress.

\section{Experimental Techniques}

All the operations were made on Xenopus laevis embryos obtained from hormonally stimulated adults and incubated at room temperature. Operated embryos were maintained in MMR solution (100 mM NaCl, $2 \mathrm{mM} \mathrm{KCl}, 2 \mathrm{mM} \mathrm{CaCl}_{2}, 1 \mathrm{mM} \mathrm{MgCl}_{2}, 5$ $\mathrm{mM}$ HEPES, $\mathrm{pH} 7,4)$. The optical histology, transmission and scanning electron microscopy techniques were routine (see Beloussov et al., 1990, 2000). For modifying tensile stresses, the following approaches were used:

1. Relaxation of mechanical stresses either by localized incisions, or by making a radial cut through embryonic body and inserting a piece of a homologous tissue within the wound gap (Fig. 6A; see Beloussov et al., 1990 for more details).

2. Artificial stretching of embryonic tissues by two 70 - to $80 \mu \mathrm{m}$ diameter glass needles attached to the agarose substrate (prepared from $2 \%$ agarose). We used either 1 -fold stretching, extending a sample up to about $150 \%$ of its initial length, or 2 -fold stretching (the both sets of stretching separated by a 7-10 $\mathrm{min}$ time period). In the last case the overall stretching reached $200 \%$ of the initial sample's length.

For labeling embryonic tissues fluorescein-dextran (40000 MW, "Mol. Probes"), were injected into either two dorso-medial or two ventro-medial blastomeres at 32 blastomeres stage (10 ng per blastomere). Fluorescence under UV excitation was monitored with the use of an Olympus SZX9 epifluorescent microscope with fluorescent block U-RFL-T.

\section{Acknowledgements}

The authors thank Dr $R$. Gordon for critically reading the manuscript and improving English language. This work was supported by the Russian Fund for Fundamental Research, grant \# 05-04-48681.

\section{References}

BELOUSSOV, L.V. (1988) Contact polarization of embryonic cells in Xenopus laevis embryos during gastrulation. Ontogenez (Sov. J. Dev Biol.) 19: 48-54.

BELOUSSOV, L.V., ERMAKOV A.S. (2001) Artificially applied tensions normalize the development of the relaxed Xenopus laevis embryos. Ontogenez (Russ J. Dev. Biol 32: 288-294.

BELOUSSOV, L.V. and GRABOVSKY, V.I. (2006). Morphomechanics: goals, basic experiments and models. Int. J. Dev. Biol. 50: 81-91.

BELOUSSOV, L.V., LAKIREV A.V., NAUMIDI, II and NOVOSELOV, V.V. (1990). Effect of relaxation of mechanical tensions upon the early morphogenesis of Xenopus laevis. Int. J. Dev. Biol. 34: 409-414.

BELOUSSOV, L.V., LUCHINSKAIA, N.N. and STEIN, A.A. (2000). Tension-dependent collective cell movements in the early gastrula ectoderm of Xenopus laevis embryos. Dev. Genes Evol. 210: 92-104.

BELOUSSOV, L.V., PETROV, K.V. (1983). Role of cell interactions in the differentiation of the induced tissues of amphibian embryos. Ontogenez (Sov. J.Dev Biol.) 14: 21-29.

DAVIDSON, L.C., HOFFSTROM, B.G., KELLER R. and D.W. DESIMONE (2002). Mesendoderm extension and mantle closure in Xenopus laevis gastrulation: combined roles for integrin $\alpha 5 \beta 1$, fibronectin and tissue geometry. Dev. Biol. 242: 109-129.

ERMAKOV, A.S. and BELOUSSOV, L.V. (1998). Variability and asymmetry of the axial rudiments in Xenopus laevis embryos under the disturbance of cell movements and tension fields in the marginal zone of early gastrula. Russ. J. Dev. Biol. 29: 38-46.

GERHART J. and R. KELLER (1986). Region-specific cell activities in amphibian gastrulation. Int. Rev. Cell. Bio/2: 201-229.

GLAGOLEVA, N.S., BELOUSSOV, L.V., STEIN, A.A., LOOUCHINSKAIA, N.N. (2003) Quantitative investigation of regional and stage-specific reactions of Xenopus laevis embryonic tissues to mechanical loading. Russ J. Dev Biol. 34: 292-300.

GORDON R. (1999) The Hierarchical Genome and Differentiation Waves: Novel Unification of Development, Genetics and Evolution. World Scientific \& Imperial 
College Press, Singapore \& London.

HARDIN, J and R. KELLER (1989). The behavior and function of bottle cells during gastrulation in Xenopus laevis. Development 103, 211-230.

HOLTFRETER, J. (1944). A study of the mechanics of gastrulation. Part II. J. Exp. Zool. 95: 171-212.

KELLER, R.E. (1978). Time-lapse cinematographic analysis of superficial cell behavior during and prior to gastrulation in Xenopus laevis. J. Embryol. Exp. Morphol. 157: 223-248.

KELLER, R.E. (1981). An experimental analysis of the role of bottle cells and the deep marginal zone in gastrulation of Xenopus laevis. J. exp. Zool. 216: 81-101.

KELLER, R.E. (1984). The cellular basis of gastrulation in Xenopus laevis: active, postinvolution convergence and extension by mediolateral interdigitation. Amer. Zool. 24: 589-603.

KELLER, R. (1987). Cell rearrangements in morphogenesis. Zool. Sci. 4: 763-779.

KELLER, R.E. and M. DANILCHIK (1988). Regional expression, pattern and timing of convergence and extension during gastrulation of Xenopus laevis. Development 103: 193-209.

KELLER, R., DAVIDSON, L.A., SHOOK, D.R. (2003). How we are shaped: The biomechanics of gastrulation. Differentiation 71: 171-205.
KELLER, R.E. and J. HARDIN (1987). Cell behaviour during active cell rearrangement: evidence and speculations. J. Cell Sci Suppl. 8: 369-393.

KELLER, R.E. and S. JANSA (1992). Xenopus gastrulation without a blastocoel roof. Dev Dynamics 195: 162-176.

NIEUWKOOP P. D. and FABER J. (1956). Normaltable of Xenopus laevis (Daudin). North-Holland, Amsterdam.

SALAZAR-CIUDAD, I., JERNVALL J and S.A. NEWMAN (2003). Mechanisms of pattern formation in development and evolution. Development 130: 2027-2037.

SCHNEIDER, S., STEINBEISSER, H., WARGA, R.M. and HAUSEN, P (1996). Beta-catenin translocation into nuclei demonstrates the dorsalizing centers in frog and fish embryos. Mech. Dev. 57: 191-198.

SPEMANN, H. (1936). Experimentelle Beitrage zu einer Theorie der Entwicklung. Jena, Fisher.

WILSON P.A., OSTER, G and KELLER, R. (1989) Cell rearrangements and segmentation in Xenopus: direct observations of cultured explants. Development 105: 155-166.

YAMADA, T. (1994). Caudalization by the amphibian organizer: brachyuri, convergent extension and retinoic acid. Development 120: 3051-3062. 